\title{
0215 INTERVENTIONS TO PREVENT SUICIDES IN SRI LANKA:
} A RANDOMISED CONTROL TRIAL

P de Silva*, D de Silva, S Jayasinghe, R de Alwis Seneviratne, M Abeyratne, D Rajapakse, N Mendis Correspondence: Ministry of Health, 18/3 Wijesekara Road, Dehiwala 001, Sri Lanka

\subsection{6/ip.2010.029215.215}

Objective To determine effectiveness of an intervention and follow-up of patients admitted following attempted suicide. Methods 300 patients admitted following attempted suicide allocated to an intervention (a session of psycho-education) and follow up or treatment as usual group using random numbers. The inclusion criteria included age above 12 years, having telephone access, and agreeing to participate in the research. Those residing in the Colombo Municipal area who did not have telephone access were given an additional option of home visits by area family health worker (FHW). Follow up was by telephone interviews or FHW visits while non-intervention subjects were traced at 18 months.

Results 668 persons were initially interviewed and 300 recruited. The intervention arm had 151 allocated of whom $96(64 \%)$ received telephone follow up and $55(36 \%)$ received FHW visits, while in the non-intervention group this was 115 (77.1\%) and $34(22.9 \%)$, respectively. At 18 months there were $32(10.6 \%)$ drop outs, with 135 in the intervention group while 132 in the non-intervention group. In the non-intervention group $39(29.5 \%)$ requested support, $35(26.5 \%)$ sought support and there were three $(2.3 \%)$ suicides, five $(3.8 \%)$ re-attempted suicides while in the intervention group these were 81 (59.3\%), $76(56.3 \%)$, one $(0.7 \%)$ and three $(2.2 \%)$, respectively.

Conclusion Intervention group requested more support and had a lower trend towards repeat suicides. Telephone and FHW follow-up is a feasible method to provide information and support. 